\title{
Effects of Influenza Vaccination on the Response to BNT162b2 Messenger RNA COVID-19 Vaccine in Healthcare Workers
}

\author{
Marilena Greco ${ }^{\mathrm{a}, \mathrm{d}} \mathbb{}$, Federico $\mathrm{Cucci}^{\mathrm{b}}$, Pierfrancesco Portulano ${ }^{\mathrm{b}}$, Roberta Assunta Lazzari ${ }^{\mathrm{a}}$, \\ Cosimo Caldararo $^{\mathrm{b}}$, Fernando Sicuro ${ }^{\mathrm{a}}$, Carmelo Catanese ${ }^{\mathrm{c}}$, Giambattista Lobreglio ${ }^{\mathrm{a}}$
}

\begin{abstract}
Background: Vaccine-induced immunity is at present the main strategy to stop the spread of severe acute respiratory syndrome coronavirus 2 (SARS-CoV-2). Recent evidences suggested a protective effect of influenza vaccination against coronavirus disease 2019 (COVID-19) severity, while impact on the immune response to BNT162b2 messenger RNA (mRNA) vaccine is under investigation.

Methods: We aimed to evaluate this aspect in a cohort of 297 healthcare workers (108 males, 189 females) after seasonal influenza vaccination compared to no-flu-vaccination. VAX+ (165 individuals; 63 males and 102 females) had tetravalent influenza vaccine, and VAX(132 individuals; 45 males and 87 females) had no flu vaccination. Anti-spike-receptor binding domain (RBD) level was tested 15 - 70 days after BNT162b2 second inoculum.
\end{abstract}

Results: Increased antibody response was observed in total VAX+ compared to VAX- (2,047.4 vs. 1,494.2 binding antibody unit (BAU)/ $\mathrm{mL}, \mathrm{P}=0.0039)$, independently from gender and body mass index (BMI). Younger total individuals $(<35$ years) showed significant increase of the level of binding antibodies (2,184.8 vs. 1,590.9 BAU/ $\mathrm{mL}, \mathrm{P}=0.0038$ ) compared to $\geq 35$ years; young/old difference was lost restricting to $\mathrm{VAX}+$ subgroup. Flu vaccinations appear associated to better antibody response in older individuals $(\mathrm{P}=0.027, \geq 35$ years VAX+ vs. VAX-). A decreasing trend during time was observed for both VAX+ and VAX-, except for $<35$ years VAX- individuals. Early response was higher in VAX+ compared to VAX-; however a more rapid waning was observed in $\mathrm{VAX}+$ subjects.

Conclusions: Our data showed better antibody response to SARS-

Manuscript submitted August 30, 2021, accepted September 29, 2021

Published online December 28, 2021

${ }^{a}$ Clinical Pathology and Microbiology, Vito Fazzi General Hospital ASL-Lecce, Lecce, Italy

bNursing Science University of Bari at Vito Fazzi General Hospital ASL-Lecce, Lecce, Italy

'Intensive Care Unit, Vito Fazzi General Hospital ASL-Lecce, Lecce, Italy ${ }^{\mathrm{d} C}$ Corresponding Author: Marilena Greco, Clinical Pathology and Microbiology, Vito Fazzi General Hospital ASL-Lecce, 73100 Piazza Muratore, Lecce, Italy. Email: grecomarilena@gmail.com

doi: https://doi.org/10.14740/jocmr4590
CoV-2 vaccine in subjects already vaccinated against seasonal influenza; this may represent one of the mechanisms underlying the cross-protective effects of influenza vaccination against heterologous infections reported in recent epidemiological studies.

Keywords: Vaccine-induced immunity; Influenza vaccination; SARS-CoV-2; BNT162b2 mRNA vaccine

\section{Introduction}

Severe acute respiratory syndrome coronavirus 2 (SARSCoV-2) infection responsible of coronavirus disease 2019 (COVID-19) disease pandemic represents the most important public health issue from December 2019 as of July 2021, and is responsible of more than 4 million deaths [1].

Vaccine-induced population immunity is at present the main strategy of primary prevention adopted to stop the spread of SARS-CoV-2.

BNT162b2 (Pfizer-BioNTech, Comirnaty) is a COVID-19 vaccine containing nucleoside-modified messenger RNA (mRNA) encoding the SARS-CoV-2 spike (S) glycoprotein. In healthy adults, two 30- $\mu \mathrm{g}$ doses of BNT162b2 elicited high neutralizing antibodies titers and robust antigen-specific cluster of differentiation (CD) $4^{+}$and $\mathrm{CD} 8^{+} \mathrm{T}$-cell responses against spike protein antigen of SARS-CoV-2 [2, 3]. Several routine immunoassay methods have been validated for measuring antibodies to the SARS-CoV-2 spike protein receptor binding domain (RBD) (generally defined as neutralizing antibody, although serum neutralization activity assay should be performed by replication competent virus requiring a biosafety level 3 laboratory or by alternative surrogate methods [4]).

BNT162b2 vaccine is reported to be $95 \%$ effective in preventing COVID-19 disease from 7 days after the second dose [5]. Recent evidence suggests a relationship between influenza vaccine coverage and COVID-19 mortality with an inverse correlation between higher influenza vaccination rate and deaths from COVID-19 [6, 7]. Jehi et al [8] developed and validated a COVID-19 infection prediction model, in which influenza vaccinated subjects had a reduced COVID-19 infection risk.

Vaccination against influenza is encouraged to facilitate the management of respiratory outbreaks coinciding with the 
peak flu season, helps in the differential diagnosis of viral-mediated adult respiratory distress and reduces hospitalization, thus allowing a more efficient use of healthcare resources [9]. There are many possible explanations for benefits of influenza vaccination in SARS-CoV-2 infection.

One possible explanation for the potential protective effect of influenza vaccination against COVID-19 is trained innate immune memory that would prime the local lung immune system for a rapid response, and that could impact acquisition of SARS-CoV-2 infection or COVID-19 disease course. As reported by Mantovani et al [10], exposure to selected vaccines, such as bacille Calmette-Guerin (BCG) or microbial components, can determine a training of innate immunity due to an increase of its baseline tone and the triggering pathogen-agnostic antimicrobial resistance. Such training appears directly related to resistance against infectious diseases, including COVID-19. Recently, Laval et al [11] drew attention to the role of durable innate immune memory conferred by myeloid cells (monocytes, macrophages and neutrophils) after the immune challenge, able to establish cryptic chromatin marks in gene regulatory regions for myeloid lineage commitment and innate immunity that became more highly expressed upon a secondary bacterial challenge thus conferring protection to the host.

A recent study [12] evaluated the impact of prior influenza and pneumococcal vaccines on humoral and cellular response to SARS-CoV-2 (BNT162b2 vaccination on 710 health care workers); at multivariable analysis a significant increase in micro-neutralization titer against SARS-CoV-2 was observed in workers receiving a concomitant influenza and pneumococcal vaccination or only influenza vaccine; female workers showed a higher increase in serum levels of anti-spike-RBD antibodies compared to male workers. Although the molecular basis of this and others results $[13,14]$ are yet unexplained, influenza/ pneumococcal vaccination seems to have a substantial impact on immunity against SARS-CoV-2 and on severity and mortality of COVID-19.

In the present study we evaluated antibody response to BNT162b2 mRNA COVID-19 vaccine in a cohort of healthcare workers (HCWs), negative for actual or previous SARSCoV-2 infection, who underwent seasonal influenza vaccination $(\mathrm{VAX}+)$ compared to subjects not immunized for seasonal influenza (VAX-). The aim of the study was to investigate differences in vaccine response between VAX+ and VAX- in individuals undergoing BNT162b2 mRNA COVID-19 vaccination.

\section{Materials and Methods}

This study included a series of 297 HCWs of the Vito Fazzi Hospital of Lecce, Italy, who underwent complete vaccination with both doses of BNT162b2 vaccine (Pfizer-BioNTech, Comirnaty) between the end of December 2020 and April 2021; all subjects underwent the second vaccine administration after 21 days from the first dose. All subjects underwent periodical nasopharyngeal swab testing (every 2 - 3 weeks) to exclude SARS-CoV-2 infection. Average age of analyzed individuals was $42 \pm 0.8$ years old, 108 males and 189 females, all enrolled individuals were healthy, non-smokers/non-drinkers, and did not display any concomitant relevant or severe comorbidity (including diabetes, hypertension, dyslipidemia, kidney disease or cancer). Subjects with relevant or severe comorbidity have been excluded; anthropometric parameters were collected for body mass index (BMI) evaluation (Table 1). A total of 165 subjects were vaccinated between October and November 2020 for seasonal influenza with tetravalent cell-based inactivated influenza vaccine (Flucelvax (Seqirus, Inc., Maidenhead, UK)).

Serum level of total antibodies (including immunoglobulin $\mathrm{G}(\mathrm{IgG})$ ) against the RBD of the spike protein of the SARS$\mathrm{CoV}-2$ was quantified by validated electrochemiluminescence immunoassay (ECLIA, Elecsys, Roche Diagnostics GmbH, Mannheim, Germany) according to manufacturer directions. Analyses were performed on Roche Cobas C8000 and results expressed as binding antibody unit $(\mathrm{BAU}) / \mathrm{mL})$, referred to the World Health Organization (WHO) International Standard for anti-SARS-CoV-2 immunoglobulin [15] certificated by WHO Expert Committee on Biological Standardization (ECBS).

The Elecsys anti-SARS-CoV-2 spike-RBD showed 99.98\% (95\% confidence interval (CI): 99.91-100\%) specificity on 5,991 samples and $98.8 \%$ (95\% CI: 98.1-99.3\%) sensitivity on 1,423 samples obtained 14 days or later after SARSCoV-2 polymerase chain reaction (PCR) confirmation [16]; this method showed also good concordance (92.3\%) of results with direct or surrogate test of virus neutralization. The established cut-off value is $0.8 \mathrm{BAU} / \mathrm{mL}$. Manufacturer reported a $100 \%$ correlation of SARS-CoV-2 binding antibodies quantification and in vitro neutralization test for titers equal or higher than $15 \mathrm{BAU} / \mathrm{mL}$.

All procedures undertaken in this study were in accordance with the ethical standards of the institutional and national research committee as well as the Declaration of Helsinki, and its later amendments and other comparable ethical standards; informed consent was obtained from all participants to the study. During COVID-19 pandemic and during the vaccination campaign, the Institutional Review Board of the Vito Fazzi Hospital authorized all research studies on this topic to give insight on this rapidly evolving disease. HCWs were requested to give preliminary informed consent for studies conducted in this context.

\section{Statistics}

Results are reported as means with standard deviation. The Student's $t$-test was used for comparison between patients and controls; statistically significant differences were established according to $\mathrm{P}$ value $(<0.05)$. Simple regression analysis was used for describing the relationship between variables. Statistical analysis was performed by MedCalc v19.9.1 statistical software.

\section{Results}

Serum samples of 297 HCWs (108 males, 189 females) immunized with BNT162b2 mRNA COVID-19 vaccine were analyzed from 14 to $>60$ days after second inoculum to evaluate anti-spike-RBD level; a subgroup of 165 individuals were vaccinated for seasonal influenza (subgroup VAX+: 63 males 
Table 1. Characteristic of Individuals Analyzed and Total Antibodies to the SARS-CoV-2 Spike (S) Protein Receptor Binding Domain (RBD) Response After Complete BNT162b2 Vaccination

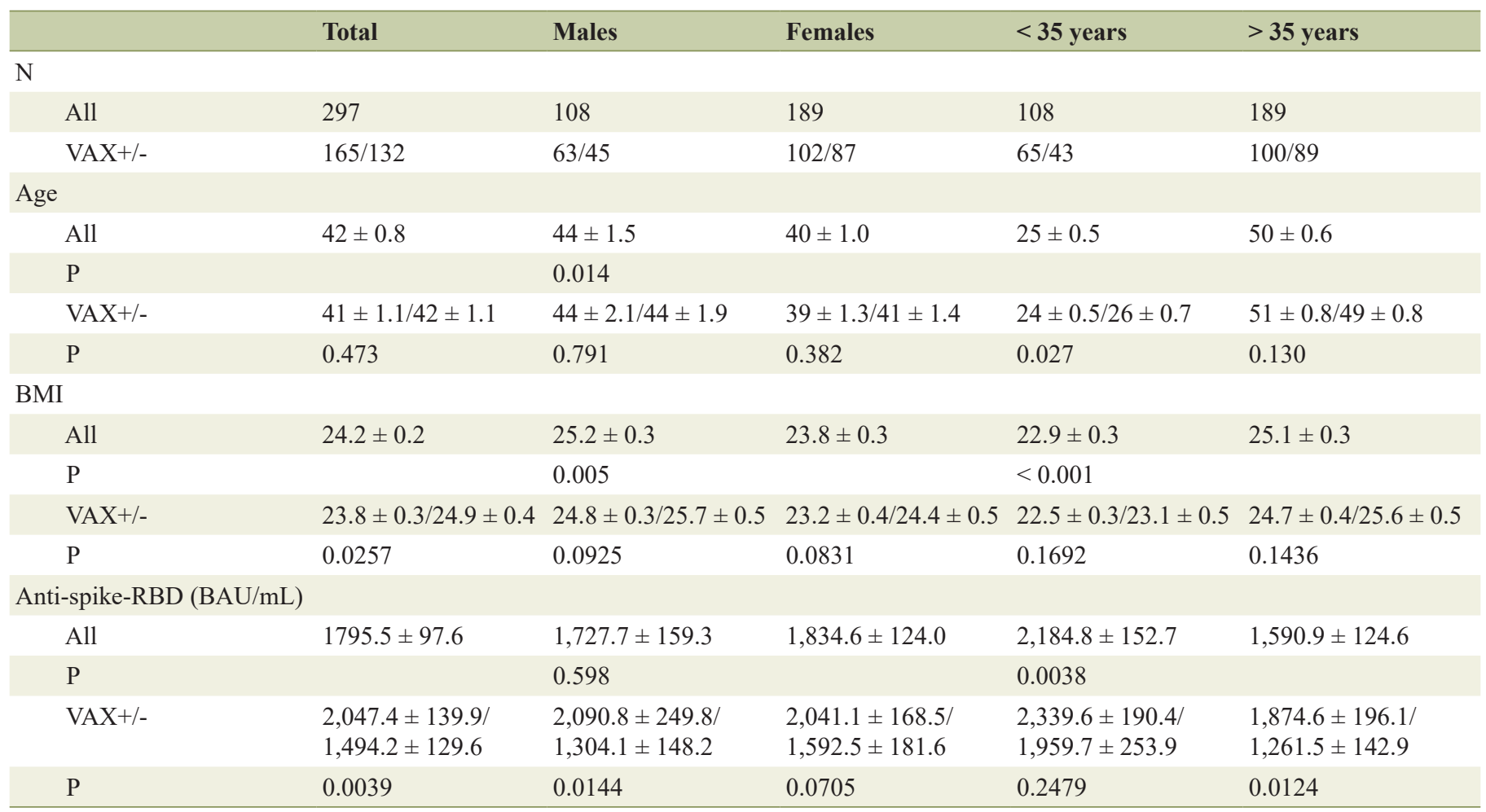

Patients with relevant comorbidity (including diabetes, hypertension, dyslipidemia, kidney disease or cancer) as well as smokers/drinkers subjects have been excluded. SARS-CoV-2: severe acute respiratory syndrome coronavirus 2; VAX+: individuals vaccinated for seasonal influenza; VAX-: individuals not vaccinated for seasonal influenza; BMI: body mass index; RBD: receptor binding domain; BAU: binding antibody unit.

and 102 females immunized by tetravalent influenza vaccine), and 132 had no flu vaccination (VAX-: 45 males, 87 females; Table1). Average age of analyzed individuals was $42 \pm 0.8$ years old; all individuals were healthy and did not display any concomitant relevant or severe comorbidity. BMI was evaluated in all subjects; we found that subjects not vaccinated for influenza had higher BMI than vaccinated ones; it was higher in males compared to females and in elderly compared to younger, independently from flu vaccination (Table 1).

We found a significant increase of total antibodies (including $\mathrm{IgG}$ ) to the SARS-CoV-2 spike protein RBD response in VAX+ individuals compared to VAX- (2,047.4 vs. 1,494.2 BAU $/ \mathrm{mL}$, with $\mathrm{P}=0.0039$; Table 1$)$. Similar values were observed by separate analysis of $\mathrm{VAX}+/$ - males and females, although it showed statistical significance only in males (VAX+ vs. VAX- was $2,090.8$ vs. $1,304.1$ with $\mathrm{P}=0.014$ in males and $2,041.1$ vs. $1,592.5$ with $\mathrm{P}=0.07$ in females). No significant difference was observed between total males and females (male to female $(\mathrm{M} / \mathrm{F}), \mathrm{P}=0.598 ; \mathrm{M} / \mathrm{F} \mathrm{VAX}+, \mathrm{P}=0.863 ; \mathrm{M} / \mathrm{F}$ VAX-, $\mathrm{P}=0.291)$.

Antibody response showed a trend of inverse correlation with BMI only in VAX- individuals, independently from gender, but not in VAX+; in total males it showed a trend of inverse correlation with BMI, not observed for total females (Fig. 1).

Average level of anti-spike-RBD antibodies was $27 \%$ higher in younger individuals. In fact, significant increased level was detected in individuals $<35$ years old compared to $\geq 35$ ( $2,184.8$ vs. $1,590.9$ BAU/mL, $\mathrm{P}=0.0038$; Table 1$)$. In accordance with literature evidences [17] we classified subjects in two groups with the criteria of 35 years old because we found significant different anti-spike-RBD antibody level between the group of age $<35$ years and both the group of 35 - 50 years old (with $\mathrm{P}=0.026)$ and $>50$ years old $(\mathrm{P}=0.006)$, whereas no significant difference was found between $35-50$ and $>50$ years old $(\mathrm{P}=0.810)$.

Among $\mathrm{VAX}+, \geq 35$ years individuals had significantly higher $(32 \%)$ antibody level compared to same age range VAX- (VAX- young/old, $\mathrm{P}=0.027$ ), while no significant difference was observed in VAX+/- young individuals (Fig. 2).

Moreover, analyzing by age, younger VAX- individuals showed a higher antibody average level compared to older $(\mathrm{P}=$ $0.024)$, while no significant difference was observed between VAX + young/old individuals.

Antibody level showed a decreasing trend during time (Fig. 3). In $\mathrm{VAX}+$ individuals this trend was evident, while in VAX $-<35$ years old individuals it was very slight; however, at the last time of observation similar values were reached in both groups. In fact, earlier immune response (i.e., antibody level measured within 20 days from complete BNT162b2 vaccination) was higher in VAX+ compared to VAX- individuals (Fig. 3c), independently from age, while later response (i.e., antibody level measured after 30 - 70days) was appreciably 

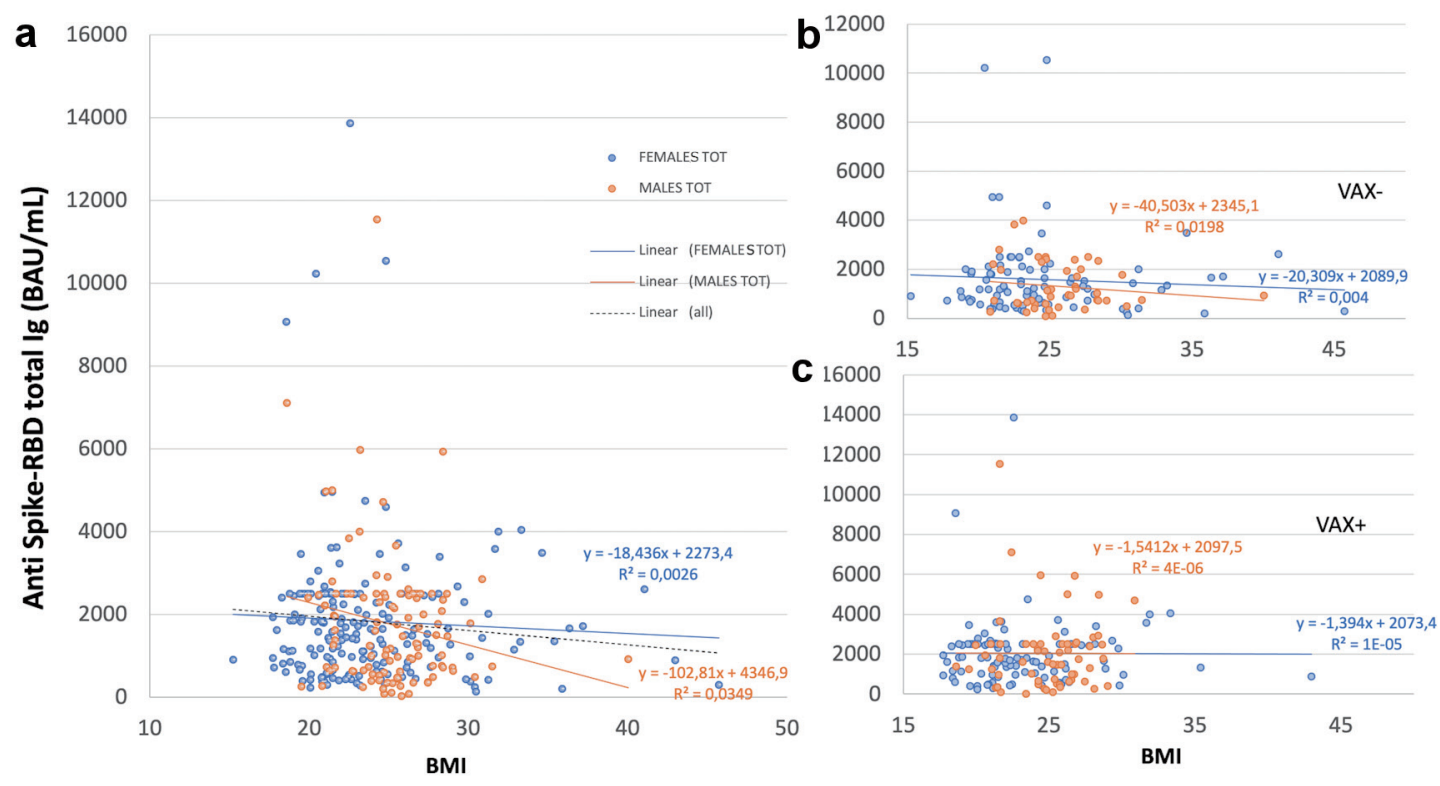

Figure 1. Correlation of total antibodies to the SARS-CoV-2 spike (S) protein receptor binding domain (RBD) and body mass index (BMI) after complete BNT162b2 vaccination in (a), all analyzed HCWs (males and females) vaccinated for seasonal influenza $(\mathrm{VAX}+)$ compared to not vaccinated for seasonal influenza (VAX-), the black dotted trend line indicates regression between all analyzed subjects; and in (b), VAX+ and (c), VAX- individuals. Simple regression analysis with $\mathrm{P}=0.054$ and 0.484 for total males and females, respectively (a); $P=0.356$ and 0.560 for VAX- males/females (b); $P=0.148$ and 0.973 for VAX+ males/females (c). SARS-CoV-2: severe acute respiratory syndrome coronavirus 2; HCWs: healthcare workers; BAU: binding antibody unit.

decreased in $\mathrm{VAX}+$ subjects compared to the initial average value, thus attaining, after 2 months from BNT162b2 vaccination, an antibody level similar to that of VAX- individuals.

\section{Discussion}

Results obtained in the present study showed the effect of influenza vaccination in a cohort of 297 healthy HCWs after double dose of BNT162b2 (Pfizer-BioNTech, Comirnaty) vac-

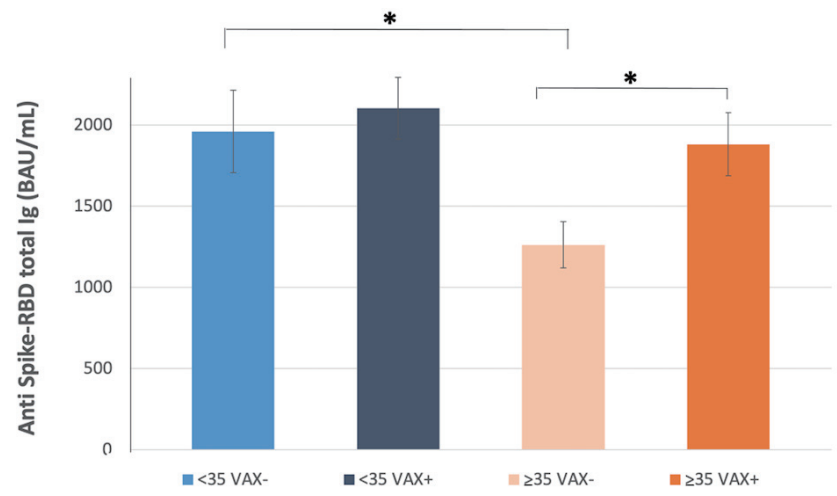

Figure 2. Total antibodies to the SARS-CoV-2 spike (S) protein receptor binding domain (RBD) response after complete BNT162b2 vaccination in individuals $<35$ years old, vaccinated for seasonal influenza (VAX+) and not vaccinated (VAX-), and individuals $\geq 35$ years old, vaccinated for seasonal influenza (VAX+) and not vaccinated (VAX-). Asterisks indicate statistical significance ( $\left.{ }^{*} P<0.05\right)$. SARS-CoV-2: severe acute respiratory syndrome coronavirus 2 ; BAU: binding antibody unit. cine on serum level of binding antibodies to the SARS-CoV-2 spike-RBD. As we known, exposure to selected vaccines (i.e., $\mathrm{BCG}$ or microbial components) determines the training of innate immunity (by epigenetic reshaping of the chromatin) which, upon secondary infection, determines more efficient response of myeloid enhancers, and better bacterial clearance and survival, including upon SARS-CoV-2 infection [10, 11, 18]. Moreover, trained myeloid cells appear more efficient to triggering adaptive immune responses. Mantovani et al [10] suggested that it would be important to ascertain whether the administration of influenza vaccine, and possibly an adjuvant, confers a heterologous mechanism of protection.

In the present study, we observed a significant difference in antibody levels between influenza VAX $+/$ - individuals $(2,047.4 \pm 139.9 / 1,494.2 \pm 129.6 \mathrm{BAU} / \mathrm{mL}, \mathrm{P}=0.0039$, Table 1), and no significant difference for gender. Previous data showed the association of different vaccines, including influenza vaccine, with decreased SARS-CoV-2 infection rates, severity and mortality of the disease [13, 14, 19-24], while the possibility of further potentiating the vaccine-induced immune response is still under debate. Puro et al [12] observed a substantial impact of earlier influenza and pneumococcal vaccines on the short-term neutralization response elicited by SARS-CoV-2 BNT162b2 vaccine in $710 \mathrm{HCW}$ sho completed vaccination. A significant increase in micro-neutralization titer against SARS-CoV-2, but not on the anti-spike-RBD and interferon (INF)-gamma response, was observed at multivariable analysis. This apparent discordance has been discussed by authors as possibly ascribed to the aged population, usually undergoing influenza and/or pneumococcal vaccines, known to have a reduced immune response. HCWs population 

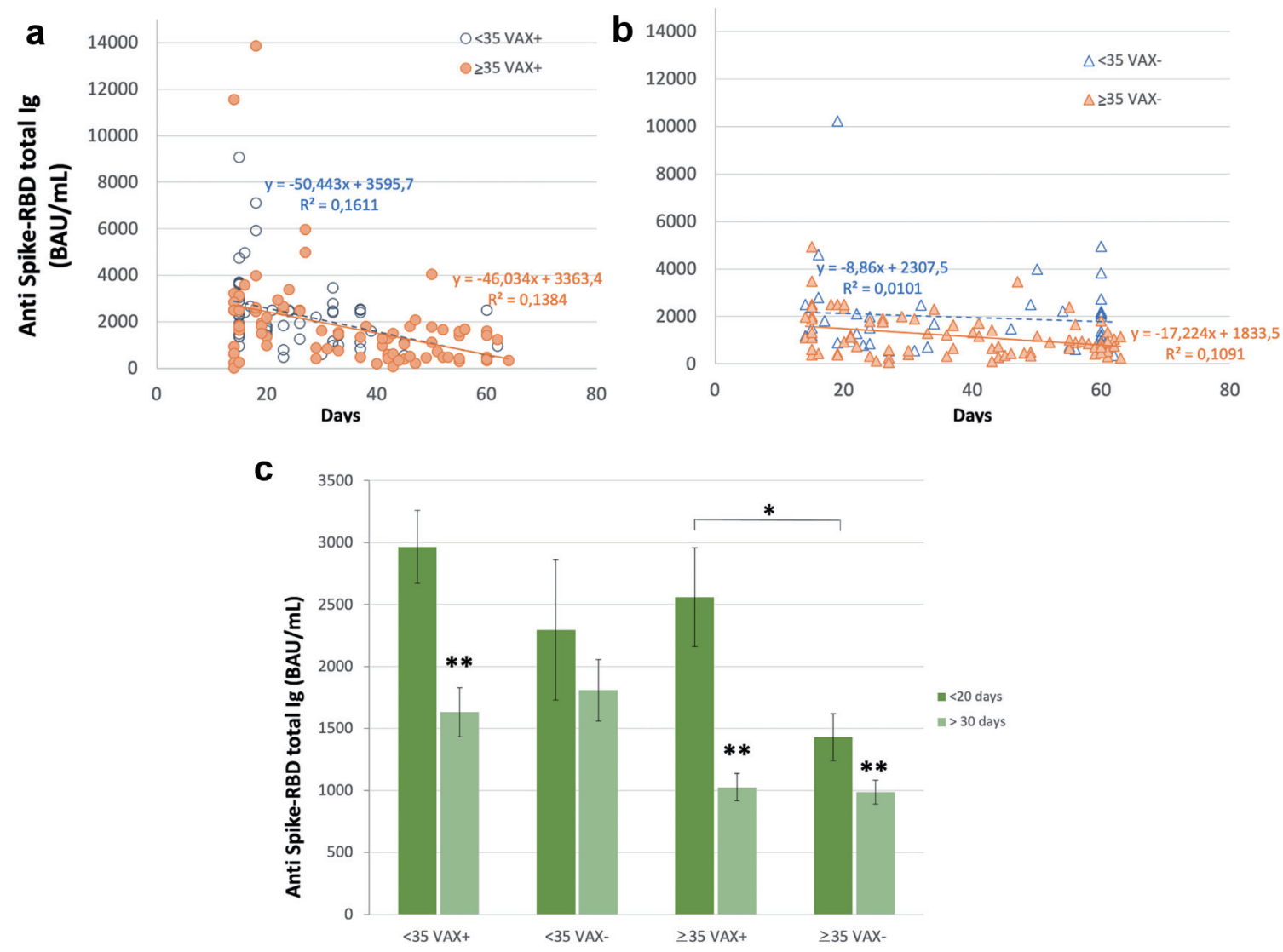

Figure 3. Serum level of total antibodies anti SARS-CoV-2 spike receptor binding domain (RBD) during time after complete BNT162b2 vaccination 15 - 70 days after second dose) in (a) individuals vaccinated for seasonal influenza $(V A X+)<35$ and $\geq$ 35 years old, simple regression analysis with $P=0.001$ and 0.0004 , respectively; (b) individuals not vaccinated (VAX-) $<35$ and $\geq 35$ years old, simple regression analysis with $P=0.0003$ and 0.003 , respectively; and (c) earlier and later immune response, measured within 20 days from complete BNT162b2 vaccination and after 30 - 70days. Asterisks indicate statistical significance $\left({ }^{*} \mathrm{P}<0.05 ;{ }^{* *} \mathrm{P}<0.01\right)$. BAU: binding antibody unit.

analyzed in the present study had lower average age $(42 \pm 0.8$ years old), and not surprising, showed a significant anti-spikeRBD increase among VAX+ individuals. We confidently assumed that the measured antibodies titers resembled the neutralization capacity because of the excellent correlation of the assay used for SARS-CoV-2 binding antibodies quantification with the in vitro neutralization test $(100 \%$ for $>15 \mathrm{BAU} / \mathrm{mL}$ according to the manufacturer).

Moreover, we found that total younger individuals had a $27 \%$ higher level of anti-sipke-RBD binding antibodies than elderly $(2,184.8 \pm 152.7$ vs. $1,590.9 \pm 124.6 \mathrm{BAU} / \mathrm{mL}, \mathrm{P}=$ 0.0038 , Table 1), independently from influenza vaccination, which conversely conferred $32 \%$ increased antibodies level in individuals older than 35 years compared to VAX- $(1,874.6 \pm$ 196.1 vs. $1,261.5 \pm 142.9 \mathrm{BAU} / \mathrm{mL}, \mathrm{P}=0.0124$; Table 1 and Fig. 2).

SARS-CoV-2 IgG antibodies has been negatively associated to BMI [25]; according to our results, it can be argued that flu vaccination reverses the decrease of SARS-CoV-2 binding antibodies level expected from increase of BMI (Table1). BMI appears to affect anti-spike-RBD levels only in VAX- population, who showed significant reduced antibody levels in indi- viduals with $\mathrm{BMI}>25(\mathrm{P}=0.0293)$; contrarily from VAX+ individuals $(\mathrm{P}=0.1887)$.

Due to the small dimension of the studied population, the result has to be confirmed in a larger population in order to have statistical significance. Among older individuals, who had significant increased BMI (25.1) than younger (22.9), we found that VAX+ individuals had significant increase of antispike-RBD than VAX- (Table 1).

We speculate that the expected reduced immune response to COVID-19 vaccine observed in older persons, who naturally have a reduced activity of the immune system, compared to young individuals, might be enhanced in presence of previous influenza vaccination in a context of antigen-nonspecific immune enhancement working in concert with, and augment, specific T- and B-cell responses. The observed increased immunologic response of younger individuals compared to elderly could be possibly ascribed to the beneficial nonspecific effects of increased immune responses towards unrelated pathogens and to the improved vaccination program to which younger individuals has been subjected from infancy by a variety of vaccines [26].

$\mathrm{VAX}+$ showed, on the short term (i.e., up to the 20th day 
after), an antibody level significantly higher than VAX- (Fig. 3); after 30 - 70 days, it rapidly decreased to similar levels in both subgroups. This unexpected or rapid waning of antibody level in $\mathrm{VAX}+$ individuals has to be further evaluated in a larger cohort of individuals due to the small number of subject so far analyzed.

In conclusion, the present study evidenced an improved humoral immune response after double dose BNT162b2 vaccine following seasonal influenza vaccination in a cohort of HCWs negative for SARS-CoV-2 infection.

Our results are in accordance with the hypothesis of a cross-protective effect of influenza vaccination against heterologous infections reported in recent epidemiological studies; a mechanism of enhanced immunization for COVID-19 vaccination could represent a benefit for some categories of patients, such as elderly adults, who lack strong immunologic responses also responsible of decreased response to immunization [18].

\section{Acknowledgments}

None to declare.

\section{Financial Disclosure}

None to declare.

\section{Conflict of Interest}

The authors declare no conflict of interest.

\section{Informed Consent}

Informed consent was obtained from all participants to the study. During COVID-19 pandemic and during the vaccination campaign, the Institutional Review Board of the Vito Fazzi Hospital authorized all research studies on this topic to give insight on this rapidly evolving disease. HCWs were requested to give preliminary informed consent for studies conducted in this context.

\section{Author Contributions}

MG and GL conceptualized and designed the study, analyzed the data, wrote and edited the manuscript. FC, PP, RAL and FS collected and analyzed the data. All authors approved the final article as submitted and agree to be accountable for all aspects of the work.

\section{Data Availability}

The data supporting the findings of this study are available from the corresponding author upon reasonable request.

\section{Abbreviations}

BCG: bacillus Calmette-Guerin; BMI: body mass index; ECBS: Expert Committee on Biological Standardization; ECLIA: electrochemiluminescence immunoassay; HCWs: healthcare workers; RBD: receptor binding domain; WHO: World Health Organization

\section{References}

1. World Health Organization. Coronavirus disease (COVID-19) pandemic, available at: https://www.who.int/ emergencies/diseases/novel-coronavirus-2019.

2. Walsh EE, Frenck RW, Jr., Falsey AR, Kitchin N, Absalon J, Gurtman A, Lockhart S, et al. Safety and immunogenicity of two RNA-Based COVID-19 vaccine candidates. N Engl J Med. 2020;383(25):2439-2450.

3. Sahin U, Muik A, Derhovanessian E, Vogler I, Kranz LM, Vormehr M, Baum A, et al. COVID-19 vaccine BNT162b1 elicits human antibody and TH1 T cell responses. Nature. 2020;586(7830):594-599.

4. Amanat F, Stadlbauer D, Strohmeier S, Nguyen THO, Chromikova V, McMahon M, Jiang K, et al. A serological assay to detect SARS-CoV-2 seroconversion in humans. Nat Med. 2020;26(7):1033-1036.

5. Polack FP, Thomas SJ, Kitchin N, Absalon J, Gurtman A, Lockhart S, Perez JL, et al. Safety and Efficacy of the BNT162b2 mRNA Covid-19 Vaccine. N Engl J Med. 2020;383(27):2603-2615.

6. Marin-Hernandez D, Schwartz RE, Nixon DF. Epidemiological evidence for association between higher influenza vaccine uptake in the elderly and lower COVID-19 deaths in Italy. J Med Virol. 2021;93(1):64-65.

7. Arokiaraj MC. Correlation of influenza vaccination and the COVID-19 severity (April 10, 2020).

8. Jehi L, Ji X, Milinovich A, Erzurum S, Rubin BP, Gordon S, Young JB, et al. Individualizing risk prediction for positive coronavirus disease 2019 testing: results from 11,672 patients. Chest. 2020;158(4):1364-1375.

9. Li Q, Tang B, Bragazzi NL, Xiao Y, Wu J. Modeling the impact of mass influenza vaccination and public health interventions on COVID-19 epidemics with limited detection capability. Math Biosci. 2020;325:108378.

10. Mantovani A, Netea MG. Trained innate immunity, epigenetics, and COVID-19. N Engl J Med. 2020;383(11):10781080.

11. de Laval B, Maurizio J, Kandalla PK, Brisou G, Simonnet L, Huber C, Gimenez G, et al. C/EBPbeta-dependent epigenetic memory induces trained immunity in hematopoietic stem cells. Cell Stem Cell. 2020;26(5):793.

12. Puro V, Castilletti C, Agrati C, Goletti D, Leone S, Agresta A, Cimini E, et al. Impact of prior influenza and pneumoccocal vaccines on humoral and cellular response to SARS-CoV-2 BNT162b2 vaccination. Vaccines (Basel). 2021;9(6):615

13. Candelli M, Pignataro G, Torelli E, Gulli A, Nista EC, Petrucci M, Saviano A, et al. Effect of influenza vaccine 
on COVID-19 mortality: a retrospective study. Intern Emerg Med. 2021;16(7):1849-1855.

14. Conlon A, Ashur C, Washer L, Eagle KA, Hofmann Bowman MA. Impact of the influenza vaccine on COVID-19 infection rates and severity. Am J Infect Control. 2021;49(6):694-700.

15. Mattiuzzo, et al. Establishment of the WHO international standard and reference panel for anti-SARS-CoV-2 antibody. 2020, WHO Expert Committee on Biological Standardization. WHO/BS/2020.2403.

16. Poljak M, Ostrbenk Valencak A, Stamol T, Seme K. Head-to-head comparison of two rapid high-throughput automated electrochemiluminescence immunoassays targeting total antibodies to the SARS-CoV-2 nucleoprotein and spike protein receptor binding domain. J Clin Virol. 2021;137:104784.

17. Steensels D, Pierlet N, Penders J, Mesotten D, Heylen L. Comparison of SARS-CoV-2 antibody response following vaccination with BNT162b2 and mRNA-1273. JAMA. 2021;326(15):1533-1535.

18. DiNardo AR, Netea MG, Musher DM. Postinfectious Epigenetic immune modifications - a double-edged sword. N Engl J Med. 2021;384(3):261-270.

19. Pawlowski C, Puranik A, Bandi H, Venkatakrishnan AJ, Agarwal V, Kennedy R, O'Horo JC, et al. Exploratory analysis of immunization records highlights decreased SARS-CoV-2 rates in individuals with recent non-COVID-19 vaccinations. Sci Rep. 2021;11(1):4741.

20. Nachega JB, Maeurer M, Sam-Agudu NA, Chakaya J, Ka- toto PDM, Zumla A. Bacille Calmette-Guerin (BCG) vaccine and potential cross-protection against SARS-CoV-2 infection - Assumptions, knowns, unknowns and need for developing an accurate scientific evidence base. Int J Infect Dis. 2021

21. Wilcox CR, Islam N, Dambha-Miller H. Association between influenza vaccination and hospitalisation or allcause mortality in people with COVID-19: a retrospective cohort study. BMJ Open Respir Res. 2021;8(1):e000857.

22. Ragni P, Marino M, Formisano D, Bisaccia E, Scaltriti S, Bedeschi E, Grilli R. Association between exposure to influenza vaccination and COVID-19 diagnosis and outcomes. Vaccines. 2020;8:675.

23. Zanettini C, Omar M, Dinalankara W, Imada EL, Colantuoni E, Parmigiani G, Marchionni L. Influenza vaccination and COVID-19 mortality in the USA: an ecological study. Vaccines (Basel). 2021;9(5):427.

24. Lewnard JA, Bruxvoort KJ, Fischer H, Hong VX, Grant LR, Jodar L, Gessner BD, et al. Prevention of COVID-19 among older adults receiving pneumococcal conjugate vaccine suggests interactions between Streptococcus pneumoniae and SARS-CoV-2 in the respiratory tract. J Infect Dis. 2021.

25. Frasca D, Reidy L, Cray C, Diaz A, Romero M, Kahl K, Blomberg BB. Influence of obesity on serum levels of SARS-CoV-2-specific antibodies in COVID-19 patients. PLoS One. 2021;16(3):e0245424.

26. Benn CS, Fisker AB, Rieckmann A, Sorup S, Aaby P. Vaccinology: time to change the paradigm? Lancet Infect Dis. 2020;20(10):e274-e283. 